\title{
Magnetic Phase Transitions in the $\mathrm{NdFe}_{3}\left(\mathrm{BO}_{3}\right)_{4}$ multiferroic
}

\author{
G.A. Zvyagina, ${ }^{1}$ K.R. Zhekov, ${ }^{1}$ I.V. Bilych, ${ }^{1}$ \\ A.A. Zvyagin, ${ }^{2,1}$ I.A. Gudim, ${ }^{3}$ and V.L. Temerov ${ }^{3}$ \\ ${ }^{1}$ B.I. Verkin Institute for Low Temperature Physics and Engineering of \\ the National Academy of Sciences of Ukraine, Kharkov, 61103, Ukraine \\ ${ }^{2}$ Max-Planck-Institut für Physik komplexer Systeme, 01187 Dresden, Germany \\ ${ }^{3}$ L. V. Kirensky Institute for Physics, \\ Siberian Branch of the Russian Academy of Sciences, \\ 660036, Krasnoyarsk, Russian Federation
}

(Dated: November 11, 2018)

\begin{abstract}
Low temperature studies of the behavior of the sound velocity and attenuation of acoustic modes have been performed on a single crystal $\mathrm{NdFe}_{3}\left(\mathrm{BO}_{3}\right)_{4}$. Transitions of the magnetic subsystem to the antiferromagnetically ordered state at $T_{N} \approx 30.6 \mathrm{~K}$ have been revealed in the temperature behavior of the elastic characteristics. The features in the temperature behavior of elastic characteristics of the neodymium ferroborate and its behavior in the external magnetic field, applied in the basic plane of the crystal, permit us to suppose that the transition to an incommensurate spiral phase is realized in the system. This phase transition behaves as the first order one. $H-T$ phase diagrams for the cases $\mathbf{H} \| \mathbf{a}$ and $\mathbf{H} \| \mathbf{b}$ have been constructed. The phenomenological theory, which explains observed features, has been developed.
\end{abstract}

PACS numbers: 72.55.+s; 74.25.Ld

Keywords: rare earth ferroborates, magneto-elastic interaction, magnetic phase transitions 


\section{INTRODUCTION}

Rare-earth ferroborates with the common formula $\mathrm{RFe}_{3}\left(\mathrm{BO}_{3}\right)_{4}(\mathrm{R}=\mathrm{Y}, \mathrm{La}-\mathrm{Nd}$; Sm-Er $)$ are studied intensively during last years, because they possess a set of interesting optical, magnetic, and magneto-electric properties. Belonging of these crystals to the class of multiferroics makes them very perspective subjects for the creation of new multifunctional devices $[1]$.

Magnetic properties of the rare-earth ferroborates are determined by the presence there of two types of magnetic ions, which belong to $3 d$ and $4 f$ elements. The specifics of their magnetic properties is defined, first, by the behavior of the magnetic iron subsystem, and second, by the characteristic features of the electron structure of the rare-earth ion, which is formed by the crystalline electric field, and, naturally, by the $f-d$ interaction. In all compounds of this family the antiferromagnetic ordering of the iron subsystem takes place at low temperatures $\left(30 \mathrm{~K} \leq T_{N} \leq 40 \mathrm{~K}\right)$. The question about the state of the rareearth subsystem is still under debates. In the majority of papers it is supposed that the rare-earth subsystem is magnetized due to the $f-d$ interaction. The temperature of the antiferromagnetic transition weakly depends on which rare-earth ion forms the ferroborate. However, the ions $\mathrm{R}^{3+}$ bring an essential contribution to the orientation of the $\mathrm{Fe}^{3+}$ magnetic ions in the ordered state, and the magnetic anisotropy. Depending on the rare-earth ion, the compounds can be antiferromagnets of "easy-axis" or "easy-plane" type, or can be spontaneously transferred between those types. The concurrence between the contributions of two magnetic systems in the formation of the magnetic structure defines the existence of phase transitions, which take place with the change of the temperature, as well as with the change of the external magnetic field $[1,2]$.

The presence of the essential coupling between the magnetic, electric, and elastic subsystems of ferroborates yields to the onset of multiferroelectric effects, which reveal themselves most sharply in the vicinities of the spontaneous and magnetic field-induced phase transformations. This is why, the investigation of the elastic properties of such compounds in the vicinities of phase transitions is very interesting.

The present study is related to the investigation of the magneto-elastic effects in the ferroborate $\mathrm{NdFe}_{3}\left(\mathrm{BO}_{3}\right)_{4}$. 


\section{STRUCTURE, MAGNETIC, AND MAGNETOELECTRIC PROPERTIES OF THE NEODYMIUM FERROBORATE}

The crystal structure of $\mathrm{RFe}_{3}\left(\mathrm{BO}_{3}\right)_{4}$ compounds belongs to the structural type of the natural mineral huntite $\mathrm{CaMg}_{3}\left(\mathrm{CO}_{3}\right)_{4}$ that crystallizes in the space group R32 of the trigonal system. In this structure, chains of edge-sharing $\mathrm{FeO}_{6}$ octahedra run along the trigonal axis, being interconnected by isolated $\mathrm{RO}_{6}$ trigonal prisms and $\mathrm{BO}_{3}$ triangles [3]. Within this family, the members with heavy rare-earth ions from Eu to $\mathrm{Yb}$, and also Y, experience a first order structural transformation to a less symmetric P312 phase, which also belongs to the trigonal type [4]. According to the data [5] $\mathrm{NdFe}_{3}\left(\mathrm{BO}_{3}\right)_{4}$ does not undergo the symmetry reduction and remains in the $\mathrm{R} 32$ space group down to $2 \mathrm{~K}$.

Magnetic properties of $\mathrm{NdFe}_{3}\left(\mathrm{BO}_{3}\right)_{4}$ have been explored in detail by the presernt time. In particular, investigations of magnetic and thermal properties of this compound have evidenced that below the temperature $T_{N} \sim 30 \mathrm{~K}$ its' magnetic structure was antiferromagnetic with the magnetic moments of iron oriented in the basic ab-plane. Thereby, $\mathrm{NdFe}_{3}\left(\mathrm{BO}_{3}\right)_{4}$ has been presumed as a magnet with an "easy-plane" magnetization anisotropy [6, 7]. Authors of those papers have believed that the subsystem of $\mathrm{Nd}$ remained paramagnetic. The magnetic Nd moments magnetized by the effective field developed by the ordered subsystem of iron ions also lay in the ab-plane. The assumption about the existence of three kinds of domains with vectors of antiferromagnetism oriented along the $\mathrm{C}_{2}$ binary axes in zero magnetic field has brought out. On one of the domains the direction of the vector of antiferromagnetism coincides with the crystallographic a-axis. Small jumps of the magnetization in the magnetic field applied within the basic plane have been registered [7]. These peculiarities (at $H_{s f}=1 \mathrm{~T}$ and $T=2 \mathrm{~K}$ ) have been related to the spin-flop transition. As a result, both Fe and Nd moments have turned perpendicular to the direction of the external field within the basic plane [8]. The same conclusion has been obtained in [5]. The detailed analysis of the magnetically diffracted intensities as a function of the magnetic field and azimuth angle in the hard X-ray scattering experiments in $\mathrm{NdFe}_{3}\left(\mathrm{BO}_{3}\right)_{4}$ have suggested that the magnetic moments of the Fe ions have aligned in the crystallographic a-axis, leading to a domain structure formation, since there were three equivalent directions in the basic plane. On the other hand, the last neutron-diffraction results [9] have shown that the long-range magnetic order observed in $\mathrm{NdFe}_{3}\left(\mathrm{BO}_{3}\right)_{4}$ below $T_{N} \approx 30 \mathrm{~K}$ consisted of antiferromagnetic 
stacking along the $\mathbf{c}$ axis, where the magnetic moments of all three $\mathrm{Fe}^{3+}$ sublattices and the $\mathrm{Nd}^{3+}$ sublattice was aligned ferromagnetically and parallel to the hexagonal basal plane. According to [9], three magnetic Fe moments have equal magnitudes, and the angle between the magnetic Fe and $\mathrm{Nd}$ moments was zero. Moreover, the phase transition to the lowtemperature incommensurate (IC) magnetic structure has revealed at $T_{I C}=13.5 \mathrm{~K}$. The authors have supposed that in the incommensurate magnetic phase the magnetic structure of $\mathrm{NdFe}_{3}\left(\mathrm{BO}_{3}\right)_{4}$ has transformed into a long-period antiferromagnetic helix [9].

Magnetic ordering has induced a variety of interesting features in this material. Recently $\mathrm{NdFe}_{3}\left(\mathrm{BO}_{3}\right)_{4}$ have been reported to exhibit a significant magnetodielectric coupling in an external magnetic field [10]. The stepwise onset of the large magnetically induced electric polarization was found in the temperature range $4.2 \mathrm{~K}-25 \mathrm{~K}$ for fields $H_{c r} \sim 1 \mathrm{~T}$ parallel to the a-axis. However, even for the absence of the external magnetic field, in $\mathrm{NdFe}_{3}\left(\mathrm{BO}_{3}\right)_{4}$ the electric polarization distinct from zero has been observed below the Néel temperature [2]. A clear relation between the magnetoelectric and lattice properties have been shown in the magnetostriction measurements [10]. Jump-like peculiarities in the magnetostriction were also found in the same temperature and magnetic field range, where peculiarities in electrical polarization have been registered.

\section{EXPERIMENTAL SETUP}

Single crystals of $\mathrm{NdFe}_{3}\left(\mathrm{BO}_{3}\right)_{4}$ have been grown using a $\mathrm{Bi}_{2} \mathrm{Mo}_{3} \mathrm{O}_{12}$-based flux by the technique described in [11], and have reached the sizes up to 10-12 $\mathrm{mm}$. The seeds were obtained by the spontaneous nucleation from the same flux. Single crystals were green in color and had a good optical quality.

We worked with the crystal representing transparent hexagonal well faceted prism in height about of $4 \mathrm{~mm}$ in a direction, close to an axis of symmetry of the third order. Experimental samples with the characteristic sizes $\sim 1.9 \times 2.6 \times 3.6 \mathrm{~mm}$ (the sample 1) and $0.8 \times 1.2 \times 1.2 \mathrm{~mm}$ (the sample 2 ) have been prepared. The samples were oriented by means of the X-ray diffraction (the Laue method).

In the study the temperature and the magnetic field behavior of the velocities and attenuations of the longitudinal and transverse acoustic modes in the single crystal samples of $\mathrm{NdFe}_{3}\left(\mathrm{BO}_{3}\right)_{4}$ was performed. The range of the temperature was $1.7 \mathrm{~K}-300 \mathrm{~K}$, and the 
magnetic field was used up to $5.5 \mathrm{~T}$.

The mesurements of the relative changes of the velocity and attenuation of acoustic modes were performed using the automatized setup described in [12]. The working frequency was $54.3 \mathrm{MHz}$. The accuracy of the relative measurements with the thickness of samples $\sim 0.5 \mathrm{~mm}$ was about $\sim 10^{-4}$ in the velocity and $0.05 \mathrm{~dB}$ in the attenuation.

\section{TEMPERATURE DEPENDENCES OF THE SOUND VELOCITY AND ATTENU- ATION}

As is known, the neodymium ferroborate conserves the R32 symmetry at least up to $2 \mathrm{~K}$ [5]. Our experiments have shown that in the temperature range $300 \mathrm{~K}-30 \mathrm{~K}$ all longitudinal and transverse acoustic modes demonstrate the standard monotonic behavior without any peculiarities. However, starting from $30.6 \mathrm{~K}$ till $10 \mathrm{~K}$ the velocity and attenuation of all studied modes behave in an anomalous way.

The behavior of the transverse modes in this temperature range can be characterized as follows. At $T_{N} \approx 30.6 \mathrm{~K}$ the the jump-like decrease of the velocity is observed. For the following decrease of the temperature, the velocity continues to decrease, reaching the minimum value in the vicinity of $T \sim 25 \mathrm{~K}$. Up to $\sim 20 \mathrm{~K}$ the value of the velocity is changed non essentially. Then, one observes the essential growth of the velocity, so that at $10 \mathrm{~K}$ it returns practically to its' value at $T_{N} \approx 30.6 \mathrm{~K}$, see Figs. 1a and $2 \mathrm{a}$. We used the following notations in Figures: $\Delta s / s$ and $\Delta \alpha$ are the relative changes of the velocity and attenuation of sound waves with the wave vector $\mathbf{q}$ and the polarization $\mathbf{u}$, which are spreading along the axes $\mathbf{a}, \mathbf{b}$ and $\mathbf{c}$ of the standard for the trigonal crystal Decart system of co-ordinates (a $\left\|\mathrm{C}_{2}, \mathbf{c}\right\| \mathrm{C}_{3}$ ). The dependences for different magnetic field values are shifted for clarity along the ordinate relative to one another in Figs.1-4.

In the case of the transvwerse $\mathrm{C}_{44}(\mathbf{q}\|\mathbf{c}, \mathbf{u}\| \mathbf{a})$ the growth of the velocity starts about $T \sim 14 \mathrm{~K}$, and it is finished to $T \sim 7 \mathrm{~K}$, Fig. 3a $(H=0)$.

The behavior of the attenuation in this temperature region is seen in Figs. 1b-3b $(H=0)$. One can see that the peculiarities in the behavior correlate with the anomalies observed in the sound velocity.

The positions of the peculiarities in the velocity and attenuation near $T_{N} \approx 30.6 \mathrm{~K}$ are not changed when the direction of the temperature development. The behavior of the sound 
characteristics in the range $10 \mathrm{~K} \leq T \leq 25 \mathrm{~K}$ has the hysteresis character.

The longitudinal modes in the range $10 \mathrm{~K} \leq T \leq 30.6 \mathrm{~K}$ behave in an analogous way, though the scale of anomalies for them is an order of magnitude weaker.

From $10 \mathrm{~K}$ and up to the lowest in our experiments temperature $1.7 \mathrm{~K}$ there are no essential changes of the acoustic characteristics of the crystal.

We have found that in the discussed temperature range $10 \mathrm{~K} \leq T \leq 30.6 \mathrm{~K}$ the sample is in the inhomogeneous state (from the point of view of the sound development). The mode $\mathrm{C}_{44}$ "feels" this inhomogeneity most sharply. The sound signal below $T_{N} \approx 30.6 \mathrm{~K}$ becomes multiphase one, and the temperature dependences become jagged. Clearly seen maxima appear in the behavior of the attenuation, Fig. 4 the sample 1 . Those maxima, most probably, have the interference nature, since the change of the working frequency for $\pm 1 \mathrm{MHz}$ causes to the redistribution of their intensities and changes their temperature position for 1-2 K, Fig. 4b.

We managed to obtain the better result (to exclude the influence of the interference) by using of the significant change of sizes, and to some extend, of the shape of the sample. In the sample 2 the sound signal was practically single-phase one in all the range of temperatures, including the region of the inhomogeneity $10 \mathrm{~K} \leq T \leq 30 \mathrm{~K}$. The dependences of the sound velocity and sound attenuation appeared to be smooth, Fig. 4, the sample 2. The transverse modes, which wave vector lies in the basic plane ab "feel" the inhomogeneous state of a sample weaker. The temperature dependences of the acoustic characteristics of the samples 1 and 2 are weakly different from each other.

The magnetic field $H \leq 1 \mathrm{~T}$, applied to the basic plane $(\mathbf{H}\|\mathbf{a}, \mathbf{H}\| \mathbf{b})$. practically does not change the position of the feature at $T_{N} \approx 30.6 \mathrm{~K}$ and of the low-temperature wings of the temperature dependences of the velocity and attenuation. However, the scale of anomalies becomes significantly smaller, so that in fields $H>1 \mathrm{~T}$ only features at $T_{N}$ can be distinguished, Figs. 2,3.

Now, let us compare our results with the data for the observed specific heat, magnetic susceptibility, X-ray and neutron scatterings. As it follows from [5-9], $\mathrm{NdFe}_{3}\left(\mathrm{BO}_{3}\right)_{4}$ below the Néel temperature becomes antiferromagnetically odered with the anisotropy of the "easyplane" type (the commensurate magnetic phase [9]). The onset of a domain structure is possible in that phase.

With the following decrease of the temperature, as it was shown in $[9,13]$, the compound 
transfers to the incommensurate (IC) magnetic phase; as a result the magnetic structure is transformed into a long-period antiferromagnetic helix. The temperatures of such a phase transition, according to the neutron scattering data, performed on the powder and singlecrystal samples, are determined as $T_{I C} \approx 19 \mathrm{~K}$ and $T_{I C} \approx 13.5 \mathrm{~K}$, respectively. At the same time, the authors of study used the nonresonant X-ray magnetic scattering [5] believe that the transition to the magnetic spiral structure is not happened up to $2 \mathrm{~K}$ : Only antiferromagnetic domain structure is formed.

It is known also [2] that below $T_{N} \approx 30 \mathrm{~K}$ the spontaneous electric polarization appears in $\mathrm{NdFe}_{3}(\mathrm{BO} 3)_{4}$. The temperature dependences of the polarization along $\mathbf{a}$ and $\mathbf{b}$ axes manifest peculiarities about $17 \mathrm{~K}$, Fig. 4a.

Since there are no data about the presence of any structural transformations in $\mathrm{NdFe}_{3}(\mathrm{BO} 3)_{4}$, it is logical to relate the observed by us anomalies in the behavior of the elastic characteristics in the range $10 \mathrm{~K} \leq T \leq 30.6 \mathrm{~K}$ with the transformation of the magnetic (and electric) subsystem of the crystal. We believe that the anomalies at $T_{N} \approx 30.6 \mathrm{~K}$ are related to the transition to the magnetically ordered state. The critical temperature of this transition, which is determined from our experiments, correlates with the most frequently published value of the Néel temperature for $\mathrm{NdFe}_{3}(\mathrm{BO} 3)_{4}$.

Unfortunately, it is difficult, looking on the temperature dependences of the sound velocity and attenuation, to answer the question about the realization of the phase transition to the incommensurate magnetic phase in the system. In the temperature dependeces below $T_{N}$ there is no well-localized peculiarity, which can be defined as the temperature of the phase transition. However, for all modes in the range $10 \mathrm{~K} \leq T \leq 20 \mathrm{~K}$ we registrate the anomalous increase of the velocity, which tells us about the distortions of the crystal lattice. Besides, the temperature dependences of the characteristics of the $\mathrm{C}_{44}$ mode, observed in the massive sample 1 , have relatively sharp $(\sim 1.5 \%)$ step of the velocity, and associated with it maximum in the attenuation namely at $13.5 \mathrm{~K}$. In the thick sample 2 these anomalies become more smooth, "smeared out" in temperature, Fig. 4. In our opinion, the most probable reason for such a behavior, is the transition to the spiral magnetic state.

One can present the following arguments in favor of such an assumption. Pay attention that the temperature of the phase transition to the incommensurate state differs strongly for the powder and single-crystal samples ( $\sim 19 \mathrm{~K}$ and $\sim 13.5 \mathrm{~K}$, respectively). It is known that the reduction of the crystal, when obtaining the powder sample, brings additional me- 
chanic strains. Those strains can significantly change the temperatures of phase transitions. Besides, the transition itself can be "smeared out" for some temperature range [14, 15]. Multiferoics are especially sensitive to such external effects; there phase transitions are related to all their subsystems: magnetic, electric, and elastic ones.

We performed measurements in single crystal samples. However, in our experiments the samples cannot be considered as totally "free". They were positioned between two piezoelectric transducers (made of litium niobate), the acoustic contact with the latter was provided with the help of a thin ( 1-2 mkm) layer of an organic silicone polymer oil GKZh94. The feezing of the oil at $120 \mathrm{~K}$ may introduce to the sample additional strains, which can, in principle, change the conditions of the development of phase transitions. Besides, strains, applied to the surfaces of the sample, can be distributed inhomogeneously in its volume (especially in the case of the large sample 1).

The transition to the magetically ordered phase at $T_{N} \approx 30.6 \mathrm{~K}$ in neodymium ferroborate, as well as the transition to the incommensurate structure, is, perhaps, sensitive to the external effects (strains). It can be one of the reasons for the existance of such a large difference between the presented in literature values for the Nëel temperature (29-33 K), obtained in various experiments. It is necessary to take into account also the onset below $T_{N}$ of the spontaneous electric polarization, which, in turn, is also very sensitive to such effects. It is known that a mechanical strain, applied to ferroelectrics, totally removed the phase transition [16].

While the transition to the magnetically orderd phase for all acoustic modes manifested itself as similar peculiarities at the same temperature, the transition to the inhomogeneous structure was "smeared out" at some temperature range. It revealed itself in the anomalous change of acoustic characteristics, which starts below $20 \mathrm{~K}$ and finishes about $10 \mathrm{~K}$. The presence of the temperature hysteresis, characteristic for the transitions to incommensurate phase, implies that it is of the first order.

Now, let us discuss the features of the behavior of the transverse $\mathrm{C}_{44}$ mode in the interval $10 \mathrm{~K} \leq T \leq 30 \mathrm{~K}$, Fig. 4. The acoustic "inhomogeneity" of the sample, which yields to the multi-phase signal of the sound, is caused, most probably, by the process of the creation of domains in the antiferromagnetic phase, which agree with $[5,9]$. We take into account also that neodymium ferroborate is, probably, the nonintrinsic ferroelectric [2], i.e. its electric polarization below $T_{N}$ is, perhaps, the consequence of the magnetic ordering, see Fig $4 \mathrm{a}$. In 
that case the process of the formation of the domain structure can be more complicated. For example, the recent paper [17] reported about the observation in $\mathrm{MnWO}_{4}$ of ferroelectric domains with the unusual property: the electric polarization was caused by magnetic spirals there. In the sample, where the domains of magnetic spirals were detected, the variety of antiferromagnetic domains was observed. It is interesting also that the domain structure was not periodic, but consisted of macroscopic regions, which differed from each other by the directions of magnetic and electric vectors. We cannot exclude that in our sample similar structures appeared, which could be the reason for the observed multi-phase sound signal.

In the general case, the type of the magnetic (electric) domain structure is essentially affected by the type of the anisotropy, the orientation of the crystal surfaces with respect to crystallographic axes, the shape and size of the crystal, and possible defects. Besides, external effects, like the change of temperature, elastic strains, etc. also affect on domains $[15,18]$.

This is why, by changing the shape and the size of a sample, and the glue area (i.e. unavoidable strains), we affected on the conditions of the formation of domains. The domain structure, which has been formed in the sample 2, probably appeared to be more homogeneous than the one in the sample 1 . The conditions of the sound spreading have become more favorable, which yielded the single-phase signal of the sound, and permitted to obtain smooth temperature dependences of the sound velocity and attenuation.

\section{MAGNETIC FIELD DEPENDENCES OF THE SOUND VELOCITY AND ATTEN- UATION}

Magnetic field dependences of the velocity and attenuation of longitudinal and transverse modes for fixed values of the temperature in the range $1.7 \mathrm{~K} \leq T \leq 32 \mathrm{~K}$ have been measured in the interval from zero to $5.5 \mathrm{~T}$. The field was applied in two directions in the basic plane: along the $\mathrm{C}_{2}$ axis, $\mathbf{H} \| \mathbf{a}$, and perpendicular to it, $\mathbf{H} \| \mathbf{b}$. The field, applied along $\mathrm{C}_{3}(\mathbf{H} \| \mathbf{c})$ has not yielded any observable changes in the elastic characteristics of the crystal.

Notice from the very beginning, that the most sharp features in the magnetic field dependences were demonstrated by the transverse modes. The effects in longitudinal modes were at least one order of magnitude smaller, thus we analyze only the behavior of transverse modes below. By the way, similar difference in the behavior of transverse and longitudinal 
modes we observed in terbium and praseodymium ferroborates [19, 20], which is, probably, the generic feature of rare-earth ferroborates.

Let us consider the case $\mathbf{H} \|$ a. At $T=1.7 \mathrm{~K}$ all studied transverse velocities show the jump at the field $H_{1} \sim 0.8 \mathrm{~T}$. The scale of all observed anomalies was $-0.3-1 \%$. The anomaly in the sound velocity is accompanied by the step-like increase of the attenuation. The peculiarities in $H_{1}$ have the hysteresis character. The example of the field dependence of the sound velocity and attenuation for the $\mathrm{C}_{44}$ mode at $1.7 \mathrm{~K}$ is presented in Fig. 5 . The value of $H_{1}$ is detemined as the average value between the positions in anomalies for the velocity (attenuation) when increasing and decreasing the value of the magnetic field. Further growth of the field yields the increase of the sound velocity (the decrease of the attenuation), which, perhaps, continues above the maximal in our experiments value 5.5 T. Such a behavior of acoustic characteristics above $H_{1}$ is also anomalous. It is, probably, the consequence of some continuous process, which takes place in the crystal. We have denoted by $H_{2}$ the value of the field, at which the sharp increase of the velocity after the jump in $H_{1}$ starts, see Fig. 5.

The increase of the temperature reveals some differences in the behavior of transverse modes. All modes can be divided into two groups, according to the type of their behavior. The first group is formed by the modes, which develop in the basic plane with the polarization along $\mathbf{c}$, and $\mathrm{C}_{44}$ mode $(\mathbf{q}\|\mathbf{c}, \mathbf{u}\| \mathbf{a}, \mathbf{b})$. The second group is made up of the modes, which wave and polarization vectors lie in the basis plane (the mode $\mathrm{C}_{66}$ and the mode $\mathbf{q} \| \mathbf{b}$, $\mathbf{u} \| \mathbf{a})$.

Let us consider the characteristic features of the temperature evolution of the magnetic field behavior of the groups of both kinds, using as the example $\mathrm{C}_{44}$ and $\mathrm{C}_{66}$ modes. The dependences for different temperatures are shifted for clarity along the ordinate relative to one another in Figs.6-8.

Mode $\mathrm{C}_{44}$ (Fig. 6):

1. The feature at $H_{1}$ is reliably registered in the range $1.7 \mathrm{~K} \leq T \leq 20 \mathrm{~K}$, at that above $13 \mathrm{~K}$ it becomes hysteresis-free (line 1 ).

2. The value of the jump of the velocity at $H_{1}$ increases with the growth of the temperature in the range $1.7 \mathrm{~K} \leq T \leq 13 \mathrm{~K}$. In the range $13 \mathrm{~K} \leq T \leq 20 \mathrm{~K}$ the scale of the feature at $H_{1}$ becomes smaller. The slope of the dependences above $H_{2}$ becomes 
larger with the growth of the temperature in the range $1.7 \mathrm{~K} \leq T \leq 20 \mathrm{~K}$. The most intensive growth is observed in the range $13 \mathrm{~K} \leq T \leq 20 \mathrm{~K}$. Above $20 \mathrm{~K}$ the slope becomes smaller.

3. At temperatures $\geq 16 \mathrm{~K}$ in weak fields $(\sim 0.25 \mathrm{~T})$ one relatively weak jump appears, which has the hysteresis character, and which can be seen up to $30 \mathrm{~K}$ (line 1').

4. The feature at $\mathrm{H}_{2}$, almost of the same shape, is registered up to $30 \mathrm{~K}$. With the increase of temperature from $1.7 \mathrm{~K}$ till $13 \mathrm{~K}$ the feature is rapidly shifted to weaker fields (line 2), Above $13 \mathrm{~K}$ and up to $30 \mathrm{~K}$ its' position is stabilized about 0.6 T (line $\left.2^{\prime}\right)$.

Mode $\mathrm{C}_{66}$ (Fig. 7)

1. The feature at $H_{1}$ is registered in the range $1.7 \mathrm{~K} \leq T \leq 13 \mathrm{~K}$ (line 1 ). For the mode $\mathrm{C}_{66}$ the values $H_{1}$ for each temperature is about $0.1 \mathrm{~T}$ below than for $\mathrm{C}_{44}$ mode.

2. The value of the jump of the velocity at $H_{1}$ and the slopes of the curves above $H_{2}$ decrease with the growth of temperature in the range $1.7 \mathrm{~K} \leq T \leq 13 \mathrm{~K}$. About $13 \mathrm{~K}$ the features at $H_{1}$ and $H_{2}$ become less distinguishable. The velocity (and the attenuation) of the $\mathrm{C}_{66}$ mode basically do not depend on the field. Further dynamics of those anomalies in the range $13 \mathrm{~K} \leq T \leq 30 \mathrm{~K}$ is described in 4 .

3. The weak hysteresis anomaly in the velocity in fields $\sim 0.25 \mathrm{~T}$ can be seen $15 \mathrm{~K}$ $\leq T \leq 18 \mathrm{~K}$, Fig. 7b. In analogy with the $\mathrm{C}_{44}$ mode we have denoted that region as the line 1'.

4. The feature at $H_{2}$ is registered up to temperatures $\leq 13 \mathrm{~K}$. Above $13 \mathrm{~K}$ the increase of the velocity, which we earlier associated with the feature at $H_{2}$ is observed for fields $\leq 0.3 \mathrm{~T}$ (for $\mathrm{C}_{44}$ mode it is $\sim 0.6 \mathrm{~T}$ ). In the range of temperatures $19 \mathrm{~K} \leq T \leq 29 \mathrm{~K}$ the slope of the dependences is sharply increased. The hysteresis with the loop "swing" $\sim 0.7 \mathrm{~T}$ appears. We think that the $\mathrm{C}_{66}$ mode, unlike the $\mathrm{C}_{44}$ one, in this temperature range cannot "resolve" the features, related to the lines 1' and 2'. Hence, we plotted these lines at the lower and upper boundaries of the ranges of fields, in which the sharp increase of the velocity is observed (which basically agrees with the boundaries of the hysteresis loop). 
Now let us consider the case $\mathbf{H} \| \mathbf{b}$. The features at $H_{1}$ and $H_{2}$ can be reliably registered in the temperature range $1.7 \mathrm{~K} \leq T \leq 13 \mathrm{~K}$ for all transverse modes (lines 1 and 2 of Fig. 8). Note that in this range for all modes the values of $H_{1}$ and $H_{2}$ for each temperature are about $\sim 0.15 \mathrm{~T}$ higher than in the case $\mathbf{H} \| \mathbf{a}$. For the $\mathrm{C}_{44}$ mode those features are manifested most sharply, see the inset of Fig. 8a. The dependences of the $\mathrm{C}_{66}$ mode are less prominent. In the range $13 \mathrm{~K} \leq T \leq 20 \mathrm{~K}$ the lines 1 and 2 come together and at temperatures above $20 \mathrm{~K}$ we cannot divide them. In fact, only one line survives, denoted as 1' (Fig. 8a,b), which we managed to register up to $30 \mathrm{~K}$. The position of the line 1', basically does not depend on the temperature and it is situated near $\sim 0.3 \mathrm{~T}$.

Based on the observed data we have constructed the phase $H-T$ diagrams for $\mathbf{H} \| \mathbf{a}$ and $\mathbf{H} \| \mathbf{b}$, Fig. 9. The points on diagrams are related to the positions of features in the velocity and attenuation of all studied transverse modes. The lines are guides to eye.

For the analysis of phase diagrams let us use the data of the measurements of the magnetization [7], X-ray [5], and neutron difraction [9, 13].

As we mentioned above, the transition to the incommensurate structure is under debates. The behavior of the elastic characteristics of $\mathrm{NdFe}_{3}\left(\mathrm{BO}_{3}\right)_{4}$, perhaps, permits to clarify the situation to some extend.

Before the neutron data it was believed [7] that below the Néel point the "easy-plane" antiferromagnetic collinear magnetic structure exists, which persist till $2 \mathrm{~K}$ minimum. The magnetic moments of iron ions lie in the basis plane and are oriented along the crystallografic axis a. Magnetic moments of neodymium ions lie also in the ab plane, magnetized by the field of iron. In this phase it is possible to form a domain structure, which permit three types of domains to exist with antiferromagnetic axes, directed at the angles $2 \pi / 3$ to each other.

The models of the behavior of the collinear structure in the magnetic field, applied in the basic plane, are proposed in several studies. They are very similar to each other. Let us consider one of them, e.g., from [8]. It is known that when studying the magnetic field behavior of the magnetization at $T=2 \mathrm{~K}$ weak hysteresis anomalies were observed at the field $H_{s f} \approx 1 \mathrm{~T}$ (for $\mathbf{H} \| \mathbf{a}$ and $\mathbf{H} \| \mathbf{b}$ ), which were explained as the manifestation of the spin-flop transition [7]. However, according to [8] that transition takes place according the following scenario. In the case of $\mathbf{H} \| \mathbf{a}$ in the field $H_{s f} \approx 0.7 \mathrm{~T}$ (the value, obtained from the mean-field calculations) in the domain with the axis of antiferromagnetism along 
the field direction the spin-flop transition takes place to the state with magnetic moments, almost perpendicular to the field. In two other domains with the axes of antiferromagnetism directed at the angles $\pi / 3$ to the field, both magnetic moments in each domain with different velocity turn to that flop state, see Fig. 10. The rotation is finished at fields $\approx 1.2 \mathrm{~T}$.

In the field $\mathbf{H} \| \mathbf{b}$ the domain with the antiferromagnetism axis along $\mathbf{a}$ is already in the flop phase, and its' magnetic moments turn to the field, Fig. 10. In two other domains the antiferromagnetism axes are at $\pi / 6$ to the direction of the field. Magnetic moments there with different directions also turn to the state of the spin-flop phase. At the field $\approx 0.76 \mathrm{~T}$ the system goes to the flop phase totally, with the jump.

Besides, the calculations have shown that when the temperature increases the looses of the stability of domains increase, and at $13 \mathrm{~K}$ the magnetization curve looses features, characteristic to the first order phase transition. Notice that the hysteresis of the magnetization in experimental curves is very weak even at the lowest temperature $2 \mathrm{~K}$. In the line at $10 \mathrm{~K}$ it is almost undetectable. This is why, it is difficult to determine from the behavior of the magnetization, how the spin-flop critical field depends on temperature.

On the other hand, the authors of neutron scattering experiments [9] believe that the collinear antiferromagnetic structure of single crystals $\mathrm{NdFe}_{3}\left(\mathrm{BO}_{3}\right)_{4}$ observed in the commensurate phase appears to transform to an antiferromagnetic long-period spiral below $T_{I C} \approx 13.5 \mathrm{~K}\left(T_{I C} \approx 19 \mathrm{~K}\right.$ for the powder sample [13]), and the spiral phase persists at $2 \mathrm{~K}$.

Now, let us return to our results. Note that in both cases $\mathbf{H} \| \mathbf{a}$ and $\mathbf{H} \| \mathbf{b}$ one can distinguish three temperature ranges, at the boundaries of which magneto-elastic characteristics of $\mathrm{NdFe}_{3}\left(\mathrm{BO}_{3}\right)_{4}$ change their behaviors: $1.7 \mathrm{~K} \leq T<13 \mathrm{~K}(1), 13 \mathrm{~K} \leq T \leq 20 \mathrm{~K}(2)$, and $20 \mathrm{~K} \leq T \leq 30 \mathrm{~K}(3)$.

The magnetic structure of the crystal in zero field in the range (3) is not under question. At least, the authors of papers, published in 2010 on neutron scattering [9] and on non-resonant X-ray magnetic scattering [5] think that it is the collinear "easy-plane" antiferromagnet, in which the domain structure with three types of domains is possible to be formed. Then for the description of a crystal in a magnetic field, applied in the basis plane in the range (3) we can use the scenario from [8]. Really, in the case $\mathbf{H} \| \mathbf{a}$ in the temperature range (3) we have two lines in the phase diagram: 1' and 2'. The line 1' follows the weak hysteresis jump of the velocity, that, perhaps, is related to the spin-flop transition in the domain with the antiferromagnetism axis along the field. The phase between the 
lines 1' and 2', according [8] is associated with the rotation of magnetic domains with the antiferromagnetism axes, directed at $\pi / 3$ to the flop state. Above the line 2', probably, the smooth rotation of all magnetic moments to the direction of the field (spin-flip) starts, which persists up to fields $\sim 100 \mathrm{~T}$ [7]. To remind, the line 2 ' is related to the feature at $H_{2}$. It is the value of the field, at which the increase of the velocity starts, which continues till the maximal in our experiments value 5.5 T.

For $\mathbf{H} \| \mathbf{b}$ in the temperature range (3) there is only one line 1' on the phase diagram. We think that it, according to [8], is related to the lose of stability of domains with the antiferromagnetism axes, directed at $\pi / 6$ to the field direction, and the transition of the total system to the flop state. Above the line 1' the smooth rotation of all magnetic moments to the direction of the field (spin-flip) begins. In this phase we register the continuous increase of the velocity.

Let us compare the values of critical spin-flop fields $H_{1}$ for the cases $\mathbf{H} \| \mathbf{a}$ and $\mathbf{H} \| \mathbf{b}$ in the range $(3): H_{1}(\mathbf{H} \| \mathbf{a}) \approx 0.25 \mathrm{~T}, H_{1}(\mathbf{H} \| \mathbf{b}) \approx 0.3 \mathrm{~T}$. As one can see, the relation $H_{1}(\mathbf{H} \| \mathbf{a}) / H_{1}(\mathbf{H} \| \mathbf{b})=\cos (\pi / 6)$ is fulfilled, which is the evidence in favor of the used model.

Now let us consider the range (1). If there were no transition in a spiral phase, the magnetic field behavior of elastic characteristics there would be analogous to the behavior in the range (3), i.e. differences in the behavior for $\mathbf{H} \| \mathbf{a}$ and $\mathbf{H} \| \mathbf{b}$ would exist. However, the inspection of the phase diagrams show that in (1) diagrams for $\mathbf{H} \|$ a and $\mathbf{H} \| \mathbf{b}$ basically coincide.

Suppose that the spiral phase is realized. As it is shown in [9], the spiral is the long-period antiferromagnetic helix, which propagates along the $\mathbf{c}$ axis with the moments perpendicular to it. The magnetic moments rotate of about $\pi+\gamma$ around the $\mathbf{c}$-axis between ajacent hexagonal planes that are interrelated via trigonal translations (the value of $\gamma$ increases with the decrease of temperature, and at $T=1.6 \mathrm{~K}$ one has $\gamma=2 \pi / 450)$. The behavior of such a spiral in the magnetic field has not been studied in [9]. However, from general grounds one can assume that in the magnetic field applied in the rotation plane, the spiral-plane flop transition can be realized (the analog of the spin-flop transition in collinear antiferromagnets). By the way, similar situation was described in the recent paper [21].

The angle of rotation of the magnetic vectors in the spiral is weak, thus the spiral-flop transition must not be critical to the field direction in the basis ab-plane of the crystal. 
Really, comparing, e.g., the magnetization dependences on the magnetic field at $2 \mathrm{~K}$ for $\mathbf{H} \| \mathbf{a}$ and $\mathbf{H} \| \mathbf{b}$ from [7], one can see that they are of the same kind.

The behavior of the elastic characteristics in the interval (3) is also practically undistinguishable for the cases $\mathbf{H} \| \mathbf{a}$ and $\mathbf{H} \| \mathbf{b}$. The most probable reason for it, in our opinion, is the realization of the spiral phase in a crystal.

As for the range (2), probably there the co-existence of two phases, the commensurate (collinear) and incommensurate (spiral) ones takes place. This is why, we see in phase diagrams in that range simultaneously lines, characteristic for the low- and higher-temperature phases. By co-existence of phases one can explain also the hysteresis in the temperature behavior of the sound velocity and attenuation, which we observed from $10 \mathrm{~K}$ till $20 \mathrm{~K}$.

\section{PHENOMENOLOGICAL THEORY}

To describe the observed in our experiments behavior of the magneto-elastic characteristics of the Nd-based multiferroic we use the Landau-like phenomenological theory. Let us write the free energy of the magnetic, electric, and elastic subsystems of the considered system in the form

$$
F=F_{e}+F_{m}+F_{m-e}+F_{m-e l}+F_{e l} .
$$

The first term describes the free energy of the electric subsystem,

$$
F_{e}=\frac{a_{1}}{2} \eta_{\perp}^{2}+\frac{a_{2}}{4} \eta_{\perp}^{4}+\frac{a_{3}}{2} \eta_{z}^{2}
$$

where $\eta_{z}$ and $\eta_{\perp}$ denote components of the electric order parameter, parallel and perpendicular to the $\mathrm{C}_{3}$ axis of the crystal, and $a_{1,2,3}$ are the standard coefficients of the Landau series (e.g., $a_{1} \sim\left(T-T_{e}\right)$, where $T_{e}$ is the temperature of the electric ordering). Here we consider the general situation, where the electric polarization can be caused not only by the magnetic ordering. The second term describes the free energy of the magnetic subsystem (for simplicity we limit ourselves with the two-sublattice approximation)

$$
F_{m}=\frac{1}{2 \chi_{\perp}} \mathbf{M}^{2}+\frac{1}{2 \chi_{\|}}(\mathbf{M L})^{2}-(\mathbf{M H})+F(\mathbf{L})+F_{\text {anis }},
$$

where $\mathbf{M}$ and $\mathbf{L}$ are the magnetization and the vector of antiferromagnetism (magnetic order parameter $), \mathbf{H}$ is the external magnetic field, $\chi_{\|}\left(\chi_{\perp}\right)$ denote the components of the magnetic susceptibility of the antiferromagnet, parallel (perpendicular) to the external magnetic field, 
$F(\mathbf{L})$ and $F_{\text {anis }}$ denote the isotropic part of the magnetic energy, which depends on the vector of antiferromagnetism only and the energy of the magnetic anisotropy. The next term describes the homogeneous and inhomogeneous interaction between the electric and the magnetic subsystems of the multiferroic

$$
F_{m-e}=-D(\mathbf{P}[\mathbf{M} \times \mathbf{L}])+\alpha_{i j k n} P_{i} L_{j} \nabla_{k} L_{n}
$$

where $\mathbf{P}$ is the vector of the electrical polarization, $D$ is the parameter of the homogeneous magneto-electric interaction, and $\alpha_{i j k n}$ is the tensor of the inhomogeneous magneto-electric coupling. The last two terms describe the free energy of the elastic subsystem and the magneto-elastic coupling. By writing this form of the free energy we neglect the direct interaction between the electric and elastic subsystems. In what follows we assume the week magneto-elastic coupling, as usually.

It is clear from the Landau expansion of the free energy of the electric part of the system, the spontaneous electric order parameter has the components $\eta_{z}^{0}=0$ and $\eta_{\perp}^{0}=\sqrt{-a_{1} / a_{2}} \sim$ $\sqrt{\left(T_{e}-T\right) / a_{2}}$. The spontaneous electric polarization $P \sim \eta_{\perp}$, according to the above, has only components, perpendicular to the $\mathrm{C}_{3}$ axis, in accordance with the measurements of the electric polarization in neodymium ferroborate [2].

Then we can denote

$$
\mathbf{H}_{e f f}=\mathbf{H}+D[\mathbf{L} \times \mathbf{P}],
$$

from which we see that the homogeneous part of the magneto-electric coupling renormalizes the external magnetic field $\mathbf{H}$. We know, however, that magnetic experiments reveal no spontaneous magnetization in the neodymium ferroborate [2], hence the vector of antiferromagnetism has to be parallel to the spontaneous electric polarization at least for weak external magnetic fields, i.e. the vector of antiferromagnetism has to lie in the plane ab, perpendicular to the $\mathrm{C}_{3}$ axis. It agrees with the results of the neutron scattering in the studied system [9]. It is not difficult to show using the fact that in the main approximation, neglecting the magnetic anisotropy, $\mathbf{M}=\chi_{\perp}\left(\mathbf{H}_{e f f}-x\left(\mathbf{H}_{e f f} \mathbf{L}\right) \mathbf{L}\right)$, where $x=\chi_{\perp} /\left(\chi_{\perp}+\chi_{\|}\right)$, that for the standard for antiferromagnets situation $\chi_{\perp} \gg \chi_{\|}$we can approximate the magnetic energy as

$$
F_{m} \approx F(\mathbf{L})+F_{\text {anis }}-\frac{\chi_{\perp}}{2} \mathbf{H}_{e f f \perp}^{2},
$$

where $\mathbf{H}_{e f f \perp}$ is the component of the effective magnetic field, perpendicular to the $\mathrm{C}_{3}$ axis. 
We can turn the consideration to the opposite way: let us consider the energy $F_{e}+F_{m-e}$ with $a_{2}=0$. Then, taking into account that $\eta \propto \mathbf{P}$ we can minimize the sum with respect to $\mathbf{P}$. Such a procedure immediately yields nonzero electric polarization $\mathbf{P}$, caused by the nonzero magnetic order parameter $P_{\perp} \sim\left(D / a_{1}\right)[\mathbf{M} \times \mathbf{L}]+P_{i n h}$, where the last term is caused by the inhomogeneous electro-magnetic coupling $\alpha$. Even for $\mathbf{M}=0$ the inhomogeneous coupling between electric and magnetic subsystems can cause nonzero electric polarization caused by the inhomogeneous spatial distribution of the vector of antiferromagnetism. It is, probably, the case for the neodymium ferroborate, which, probably, belongs to the class of nonintrinsic ferroelectrics [2].

According to the experiments [9] the inhomogeneous distribution of the magnetic order parameter is along $z\left(\mathrm{C}_{3}\right)$ axis. We can write the Landau series for the magnetic free energy for the components of the order parameter $L^{x}=L \cos \phi$ and $L^{y}=L \sin \phi$ in the weak magnetic field taking into account the most relevant parts of the magnetic and magnetoelectric energies as

$$
\begin{aligned}
& F_{m}+F_{m-e} \approx r L^{2}+u L^{4}+2 w L^{2} \cos 2 \phi \\
& +V^{-1} L^{2} \int d^{3} x\left[A\left(\nabla_{z} \phi\right)^{2}+\alpha P_{\perp} \nabla_{z} \phi\right],
\end{aligned}
$$

where $r=a\left(T-T_{N}\right), T_{N}$ is the Néel temperature, $a$ and $u$ are the constants of the Landau expansion, $A$ is proportional to the exchange coupling, $w=K-\chi_{\perp} H_{e f f \perp}^{2} / 4$ is renormalized due to the effective magnetic field coefficient of the magnetic anisotropy ( $K$ is the coefficient of the "bare" in-plane anisotropy observed in neodymium ferroborate [1, 2, 6-9]), and the last term in the integrand (the Lifshitz invariant [22]) is caused by the most relevant part of the inhomogeneous electro-magnetic coupling of the magnetic order parameter with the spontaneous electric polarization of the neodymium ferroborate. Here we have assumed that $L$ is homogeneous, which agrees with the results of neutron scattering experiments on single crystals [9]. Notice that in neodymium ferroborate the spontaneous polarization appears only in the magnetically ordered phase [2], hence, $T_{e}=T_{N}$. Minimizing the free energy with respect to $L$ and $\phi$ we obtain two equations (cf. [23])

$$
\begin{aligned}
& 2 L r+4 u L^{3}+A L\left(\frac{\partial \phi}{\partial z}\right)^{2} \\
& +2 \alpha P_{\perp} L \frac{\partial \phi}{\partial z}+4 w L \cos 2 \phi=0, \\
& 2 A L^{2} \frac{\partial^{2} \phi}{\partial z^{2}}+4 w L^{2} \sin 2 \phi=0 .
\end{aligned}
$$


There are several solutions of this set of equations. First, the solution with $L=0$ describes the magnetically disordered (paramagnetic) phase. Second, there is a phase with the homogeneous distribution of $\phi$ with $\sin 2 \phi=0$ and $L^{2}=\sqrt{\left[a\left(T_{N}-T\right)-2 w\right] / 2 u} \approx$ $\sqrt{a / 2 u}\left(T_{N}-T\right)^{1 / 2}$. That solution describes the homogeneous antiferromagnetic phase. The phase transition between the paramagnetic phase and the homogeneous antiferromagnetic one is of the second order, and it takes place at $T=T_{N}$. Finally, there exists the inhomogeneous for $\phi$ solution. Neglecting the effective magnetic anisotropy the solution is trivial $\phi=q_{0} z$, where $q_{0}$ is the vector of a spiral $q_{0}=\left|\alpha P_{\perp}\right| / 2 A$. For $w \neq 0$ we can write the solution as

$$
\phi(z)=\operatorname{am}\left(\frac{2 \sqrt{w / A} z}{k}, k\right),
$$

where $\operatorname{am}(x, k)$ is the Jacoby elliptic amplitude function with the parameter $0 \leq k \leq 1$, which is determined by the parameter of the effective anisotropy $w$. Substituting the solution to the free energy we can write

$$
\begin{aligned}
& F_{m}+F_{m-e} \approx r L^{2}+u L^{4}-\left|\alpha P_{\perp}\right| L^{2} \frac{\pi \sqrt{w}}{k K(k)} \\
& +L^{2} \frac{4 w}{2 k^{2}}\left(k-2+\frac{4 E(k)}{K(k)}\right),
\end{aligned}
$$

where $E(k)$ and $K(k)$ are the complete elliptic integrals of the first and the second order. Minimizing this expression with respect to $k$ we obtain

$$
\frac{k}{E(k)}=\sqrt{w A} \frac{8}{\pi\left|\alpha P_{\perp}\right|} .
$$

One can see that the phase of the order parameter can be written as the Fourier series

$$
\phi(z)=q z+\sum_{p=1}^{\infty} \frac{\sin (2 p q z)}{\cosh \left[p \pi K^{\prime}(k) / K(k)\right]},
$$

where

$$
q=\frac{\pi^{2}\left|\alpha P_{\perp}\right|}{8 A K(k) E(k)}
$$

is the vector of the spiral. We see that, as usually, the effective anisotropy causes the onset of odd harmonics. Obviously, for $w \rightarrow 0$ we have $k \rightarrow 0$, hence, $q \rightarrow q_{0}$ and $\phi \rightarrow q_{0} z$. On the other hand, the limit $k \rightarrow 1$ describes the infinitely large period of the spiral structure with $q \sim \sqrt{w} / 2 k \sqrt{A} \ln \left|4 / \sqrt{1-k^{2}}\right| \rightarrow 0$, i.e. it describes the transition from the inhomogeneous 
spiral phase to the homogeneous phase [23] with the jump of the spiral vector, i.e. of the first order. The free energy of the inhomogeneous phase can be written as

$$
F_{m}+F_{m-e} \approx r L^{2}+u L^{4}+L^{2} \frac{\alpha^{2} P_{\perp}^{2}}{16 A E^{2}(k)}\left(\frac{4 E(k) \sqrt{w A}}{\pi\left|\alpha P_{\perp}\right|}-1\right)
$$

The magnitude of the magnetic order parameter can be written as

$$
L^{2} \sim \sqrt{a / 2 u}\left(T^{*}-T\right)^{1 / 2}
$$

where $T^{*} \approx T_{N}-\left[\left|\alpha P_{\perp}\right| / 2 a\right]\left(\sqrt{w} / \sqrt{A}-\mid \alpha P_{\perp} / 2 A\right) \approx T_{N}-\left|\alpha P_{\perp}\right| \sqrt{w} / 2 a \sqrt{A}$.

In the main approximation the in-plane external magnetic field can produce the (first order, as a rule) phase transition between the homogeneous and inhomogeneous phases. By equating the free energies of the homogeneous and inhomogeneous phases we obtain, e.g., for the weak magnetic anisotropy the value of the critical field, at which the transition between the inhomogeneous and homogeneous phases takes place

$$
H_{c}=\frac{1}{\chi_{\perp}}\left(4 K-\frac{\alpha^{2} P_{\perp}^{2}}{2}\right) \approx \frac{4 K}{\chi_{\perp}}
$$

On the other hand, from the above we can see that the second order phase transition between the paramagnetic and the homogeneous antiferromagnetic phases basically does not depend (in our approximation) on the external magnetic field, which agrees with our experimental data.

Now, let us turn to the analysis of the behavior of the sound characteristics. The elastic and the magneto-elastic parts of the free energy can be written as

$$
F_{e l}+F_{m-e l}=\frac{C}{2} \varepsilon^{2}+B \varepsilon L^{2} \cos 2 \phi,
$$

where $C$ is the elastic modulus, $\varepsilon$ is the distortion, and $B$ is the magneto-elastic coupling constant. Taking into account that the magneto-elastic coupling is weak, we can write the equation for the renormalization of the elastic modulus as

$$
\begin{gathered}
\tilde{C}=C-\frac{\left(\frac{\partial^{2} F}{\partial \varepsilon \partial L}\right)^{2}}{\frac{\partial^{2} F}{\partial L^{2}}} \\
-\frac{\left(\frac{\partial^{2} F}{\partial \varepsilon \partial L \phi}\right)^{2}}{\frac{\partial^{2} F}{\partial \phi^{2}}} \\
-\frac{\frac{\partial^{2} F}{\partial \varepsilon \partial L} \frac{\partial^{2} F}{\partial \varepsilon \partial L \phi}}{\frac{\partial^{2} F}{\partial L \partial \phi}} .
\end{gathered}
$$


In the paramagnetic phase we have, obviously, $\tilde{C}=C$. In the homogeneous antiferromagnetic phase we get

$$
\begin{aligned}
& \tilde{C}=C-\frac{B^{2}}{2 u}-\frac{B^{2} L^{2}}{2 w} \\
& =C-\frac{B^{2}}{2 u}-\frac{B^{2}}{2 w} \sqrt{a / 2 u}\left(T_{N}-T\right)^{1 / 2} .
\end{aligned}
$$

Finally, in the inhomogeneous phase we obtain

$$
\tilde{C}=C-\frac{B^{2}}{2 u} .
$$

The sound velocity is proportional to the square root of the elastic modulus. This is why, the obtained theoretically temperature dependence of the behavior of the sound velocity is reminiscent to the one, observed in our experiments: The sound velocity is constant for $T>$ $T_{N}$, then it decreases with the decrease of temperature in the homogeneous antiferromagnetic phase, and, finally, it is almost constant in the inhomogeneous phase for $T<T^{*}$ with the value of the velocity lower than the one in the paramagnetic phase.

\section{CONCLUSIONS}

We have performed low-temperature ultrasound investigations of the neodymium ferroborate in the external magnetic field. Based on that investigation we can make the following conclusions.

In the temperature behavior of elastic characteristics of sound the transition to the magnetically ordered state is manifested.

The features of the behavior of elastic characteristics of the neodymium ferroborate in the external magnetic field, applied in the basic plane of the crystal permit us to suppose that the transition to the incommensurate spiral phase is realized in the system. This phase transition behaves as the first order one.

We have constructed the phase $H-T$ diagrams for $\mathrm{NdFe}_{3}\left(\mathrm{BO}_{3}\right)_{4}$ for the cases $\mathbf{H} \|$ a and $\mathbf{H} \| \mathbf{b}$.

We have constructed the phenomenological Landau-like theory, which qualitatively describes the behavior of the sound velocity as the function of the temperature and the field. The spiral phase is caused by the nonzero spontaneous electric polarization. The weak inplane anisotropy modifies the spiral structure, giving rise to the onset of odd harmonics for the spatial distrbution of the vector of antiferromagnetism. 
Acoustic "inhomogeneity" of the sample, which causes the multi-phase behavior of the signal, and which is revealed in the behavior of the transverse $\mathrm{C}_{44}$ mode in the range of temperatures $10 \mathrm{~K} \leq T \leq 30 \mathrm{~K}$ can be the evidence of the process of the creation of domains in a crystal. To answer the question whether the domain structure in the form of antiferromagnetic, and, perhaps, electric domains exists, one needs to perform additional magneto-optic investigations, analogous to [17].

\section{ACKNOWLEDGMENTS}

The authors thank V.I. Fomin and V.S. Kurnosov for the fruitful discussion of the results of our study. A.A.Z. thanks Max-Planck-Institut für Physik komplexer Systeme, Dresden, for kind hospitality. 
[1] A.N. Vasiliev and E.A. Popova, Fiz. Nizk. Temp. 32, 968 (2006) [Low Temp. Phys. 32, 735 (2006)].

[2] A.M. Kadomtseva, Yu.F. Popov, G.P. Vorob'ev, A.P. Pyatakov, S.S. Krotov, .I. Kamilov, V.Yu. Ivanov, A.A. Mukhin, A.K. Zvezdin, L.N. Bezmaternykh, I.A. Gudim, and V.L. Temerov, Fiz. Nizk. Temp. 36, 640 (2010) [Low Temp. Phys. 36, 511 (2010)]

[3] J.A. Campa, C. Cascales, E. Gutierrez-Puebla, M.A. Monge, I. Rasines, and C. Ruiz-Valero, Chem. Mater. 9, 237 (1997).

[4] Y. Hinatsu, Y. Doi, K. Ito, M. Wakeshima, and A. Alemi, J. Solid State Chem. 172, 438 (2003).

[5] J.E. Hamann-Borrero, M. Philipp, O. Kataeva, M. v.Zimmermann, J. Geck, R. Klingeler, A. Vasiliev, L. Bezmaternykh, B. Büchner, and C. Hess, Phys. Rev. B 82, 094411, (2010).

[6] N. Tristan, R. Klingeler, C. Hess, B. Büchner, E. Popova, I.A. Gudim, and L.N. Bezmaternykh, J. Magn. Magn. Mater. 316, e661 (2007).

[7] E.A. Popova, N. Tristan, C. Hess, R. Klingeler, B. Büchner, L.N. Bezmaternykh, V.L. Temerov, and A.N. Vasiljev, Zh. Eksp. Teor. Fiz. 132, 121 (2007) [J. Exp. Theor. Phys. 105, 105 (2007)].

[8] D.V. Volkov, A.A. Demidov, and N.P. Kolmakova, Zh. Eksp. Teor. Fiz. 131, 1030 (2007) [J. Exp. Theor. Phys. 104, 897, (2007)].

[9] M. Janoschek, P. Fischer, J. Schefer, B. Roessli, V.M. Meven, V. Petricek, G. Petrakovskii, and L. Bezmaternikh, Phys. Rev. B 81, 094429, (2010).

[10] A.K. Zvezdin, G.P. Vorobev, A.M. Kadomtseva, Y.F. Popov, A.P. Pyatakov, L.N. Bezmaternykh, A.V. Kuvardin, and E. Popova, Pis'ma v Zh. Eksp. Teor. Fiz. 83, 600 (2006) [JETP Lett. 83, 509, (2006)].

[11] L.N. Bezmaternykh, V.L. Temerov, I.A. Gudim, and N.A. Stolbovaya, Crystallogr. Rep. 50 (Suppl. 1), 97 (2005).

[12] E.A. Masalitin, V.D. Fil', K.R. Zhekov, A.N. Zholobenko, and T.V. Ignatova, and S.-I. Lee, Fiz. Nizk. Tem. 2993 (2003) [Low Temp. Phys. 29, 72 (2003)].

[13] P. Fisher, V. Pomjakushin, D. Sheptyakov, L. Keller, M. Janoschek, B. Roessli, J. Schefer, G. Petrakovskii, L. Bezmaternich, V. Temerov, and D. Velikanov, J. Phys.: Condensed Matter 
18, 7975 (2006).

[14] A.P. Levanyuk and A.G. Sigov, Defects and Structural Phase Transitions, Gordon and Breach, NY (1988).

[15] B.A. Strukov, Phase transitions in ferroelectric crystals with defects, Soros Educational Journal, No. 12, pp. 95-101, (1996) (in russian)].

[16] V.V. Gladkii, S.N. Kallaev, V.A. Kirikov, E.S. Ivanova, and L.A. Shuvalov, Suppression of Ferroelectricity by Low Uniaxial Stresses in some Crystals with Successive Phase Transitions, Ferroelectrics, 125, 171 (1992).

[17] D. Meier, M. Maringer, Th. Lottermoser, P. Becker, l. Bohatý, and M. Fiebig, Phys. Rev Lett. 102, $107202(2009)$.

[18] V.G. Baryakhtar and B.I. Ivanov, Modern Magnetism, Moscow, Progress, (1986).

[19] G.A. Zvyagina,, K.R. Zhekov, L.N. Bezmaternykh, I.A. Gudim, I.V. Bilych, and A.A.Zvyagin, Fiz. Nizk. Temp. 34, 1142, (2008) [Low Temp. Phys. 34, 901, (2008)].

[20] G.A. Zvyagina,, K.R. Zhekov, A.A.Zvyagin, I.V. Bilych, L.N. Bezmaternykh, and I.A. Gudim, Fiz. Nizk. Temp. 36, 376, (2010) [Low Temp. Phys. 36, 296, (2010)].

[21] H. Yamaguchi, S. Ohtomo, S. Kimura, M. Hagiwara, K. Kimura, T. Kimura, T. Okuda, and K. Kindo, Phys. Rev. B 81, 033104, (2010).

[22] E.M. Lifshitz, Zh. Eksp. Teor. Fiz. 11, 253 (1941); ibid., 11269 (1941).

[23] I.E. Dzyaloshinskii, Zh. Eksp. Teor Fiz. 47, 992 (1964) [Sov. Phys. JETP 20, 665 (1965)]. 

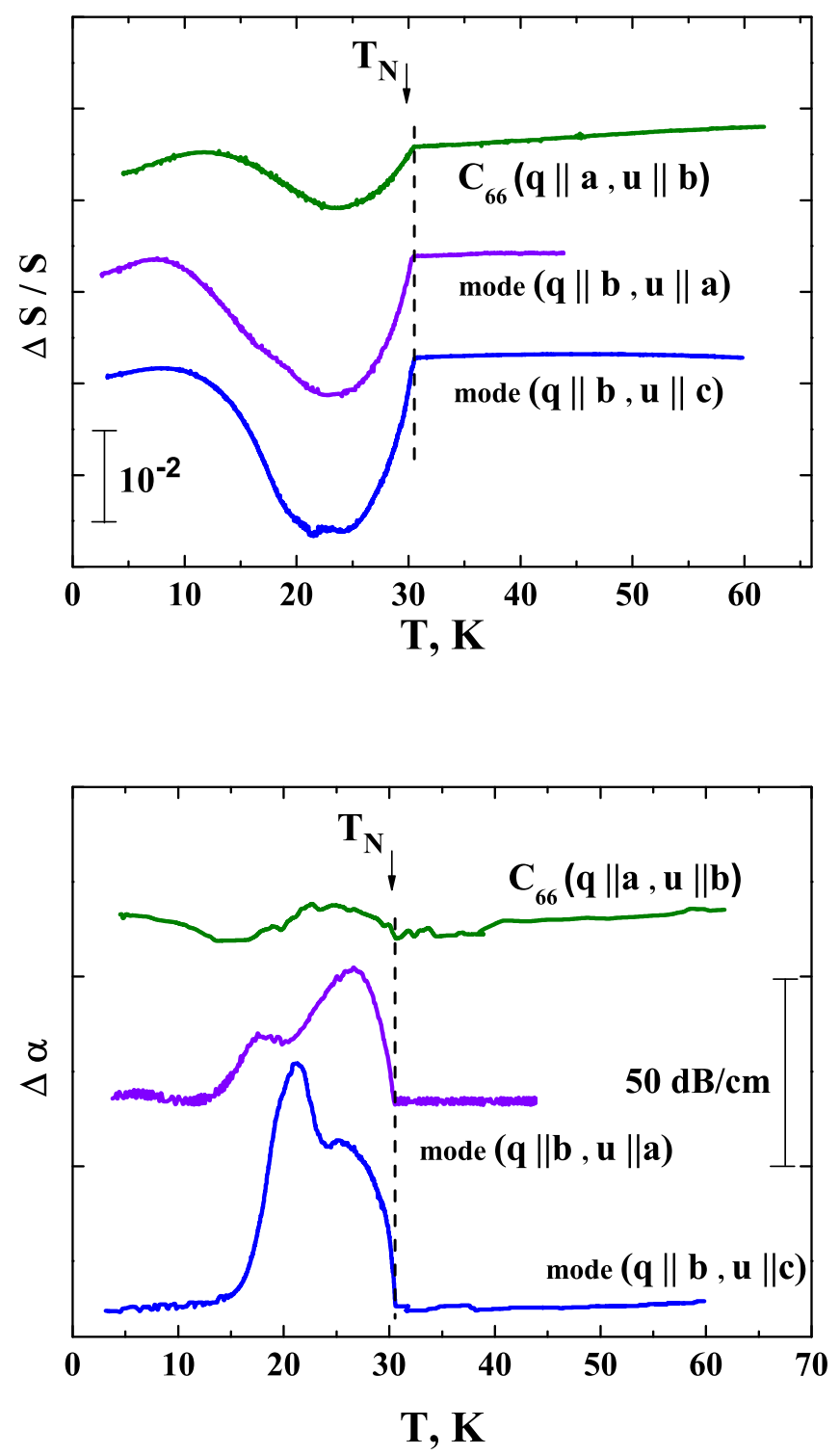

FIG. 1: (Color online) The temperature dependence of the behavior of the sound velocity (a) and attenuation (b) of the various transverse acoustic modes. 

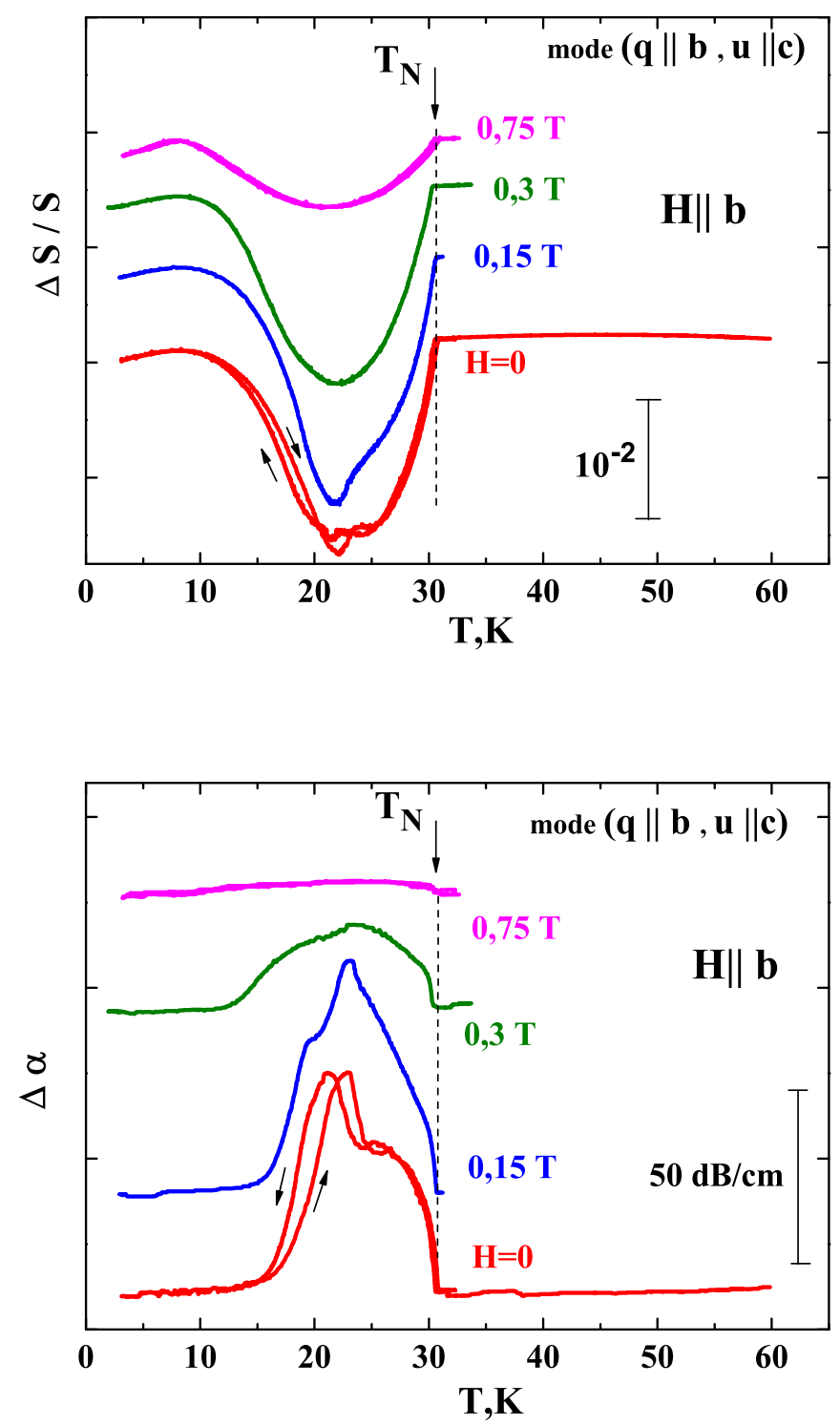

FIG. 2: (Color online)The temperature dependence of the behavior of the sound velocity (a) and attenuation (b) of the transverse mode $(\mathbf{q}\|\mathbf{b}, \mathbf{u}\| \mathbf{c})$ in the magnetic field $\mathbf{H} \| \mathbf{b}$. 

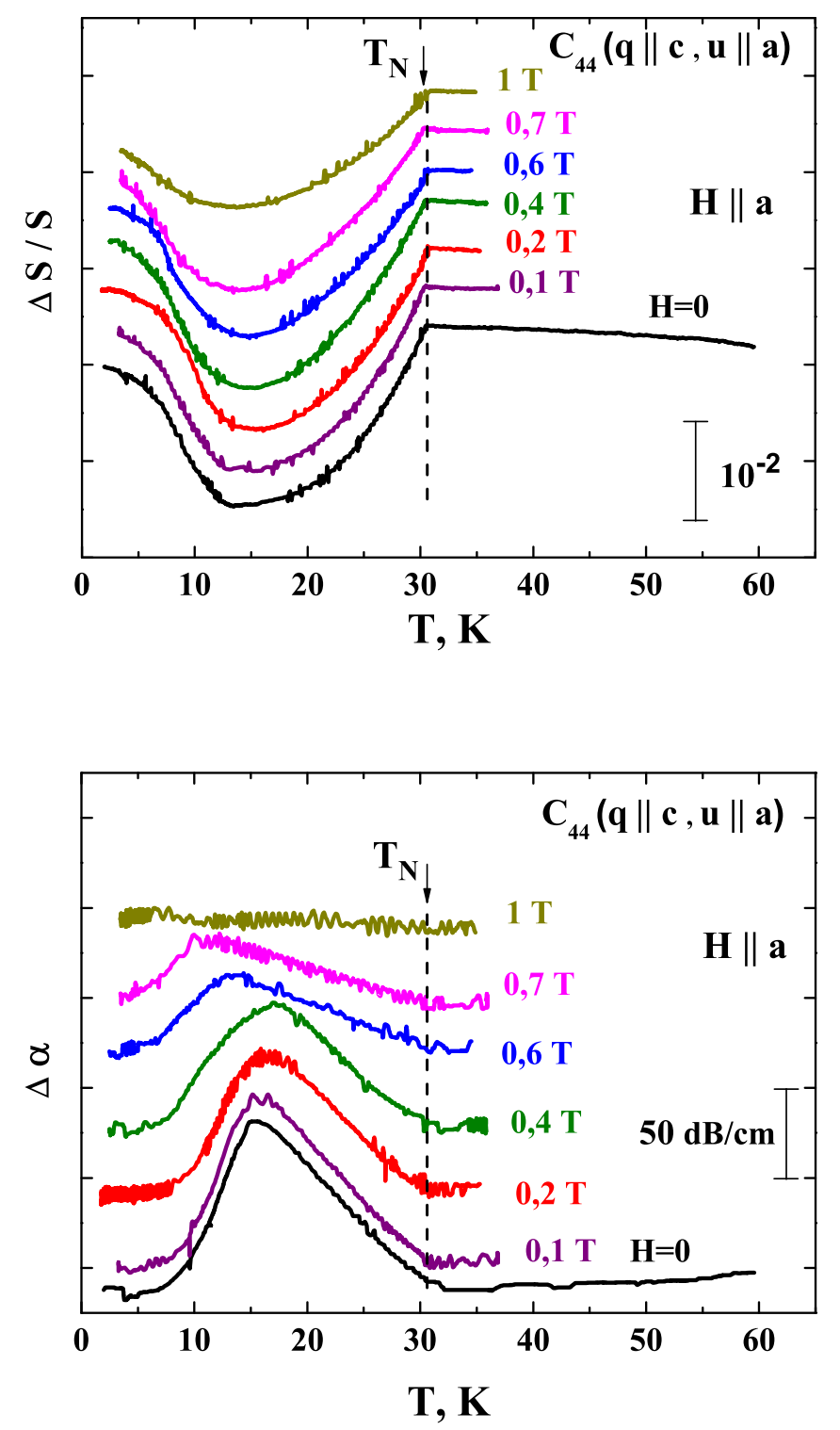

FIG. 3: (Color online)The temperature dependence of the behavior of the sound velocity (a) and attenuation (b) of the transverse mode $(\mathbf{q}\|\mathbf{c}, \mathbf{u}\| \mathbf{a})$ in the magnetic field $\mathbf{H} \| \mathbf{a}$. 

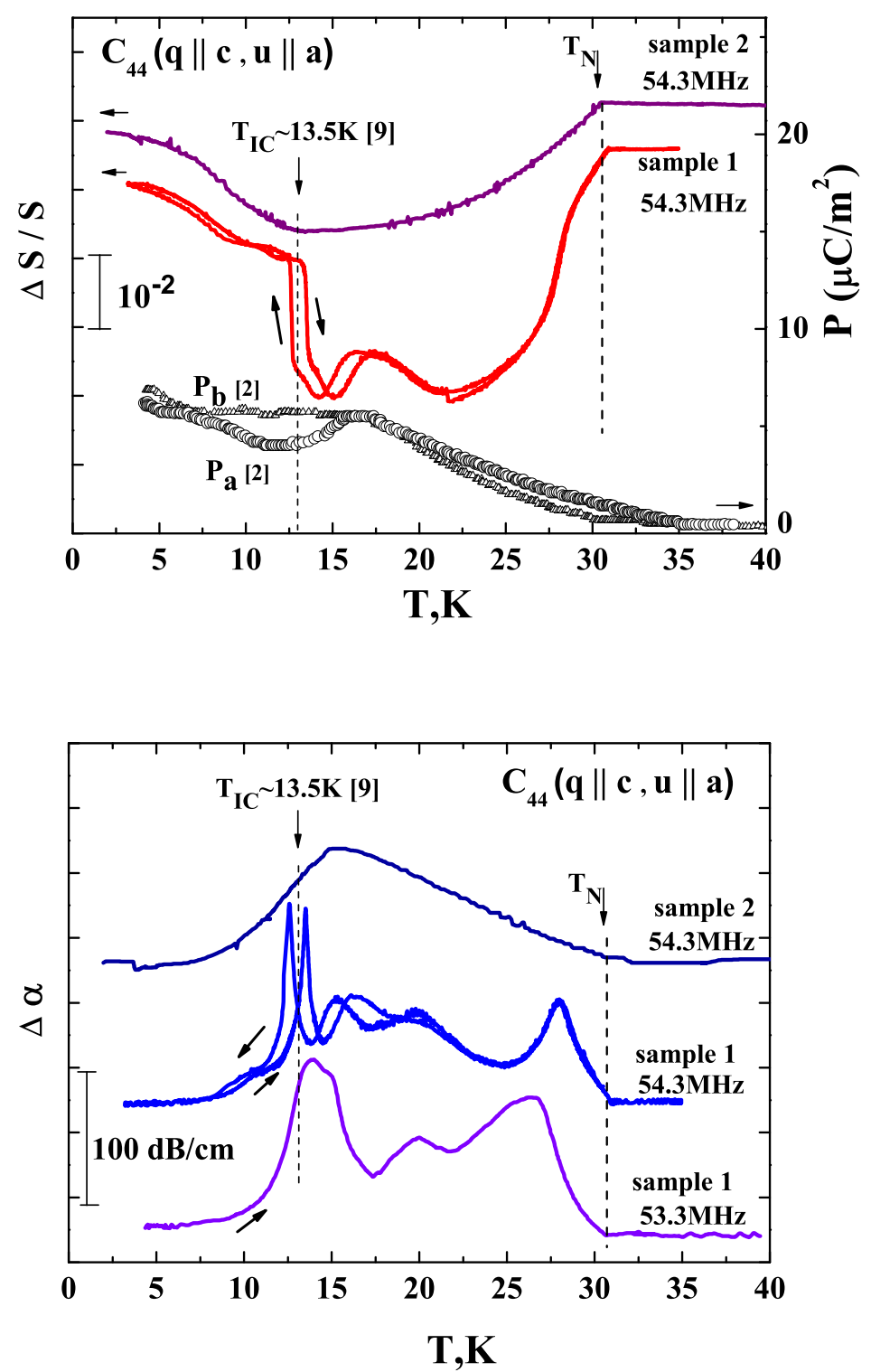

FIG. 4: (Color online)The temperature dependence of the behavior of the sound velocity (a) and attenuation (b) of the transverse mode $\mathrm{C}_{44}(\mathbf{q}\|\mathbf{c}, \mathbf{u}\| \mathbf{a})$ obtained for the samples 1 and 2. The temperature dependences of the spontaneous electric polarization [2] are also plotted. The temperature dependences of the behavior of the attenuation for the sample 2 are taken at the frequencies $54.3 \mathrm{MHz}$ and $53.3 \mathrm{MHz}$. 


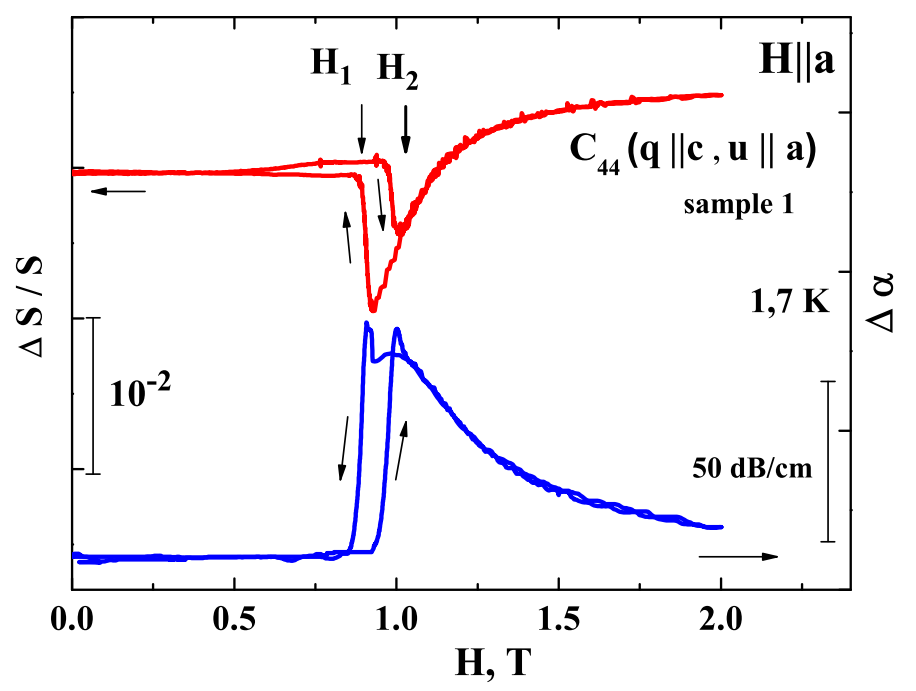

FIG. 5: (Color online) Magnetic field dependence of the behavior of the velocity and attenuation of the acoustic mode $\mathrm{C}_{44}(\mathbf{q}\|\mathbf{c}, \mathbf{u}\| \mathbf{a})$ at the temperature $1.7 \mathrm{~K}$ in the magnetic field $\mathbf{H} \| \mathbf{a}$. 

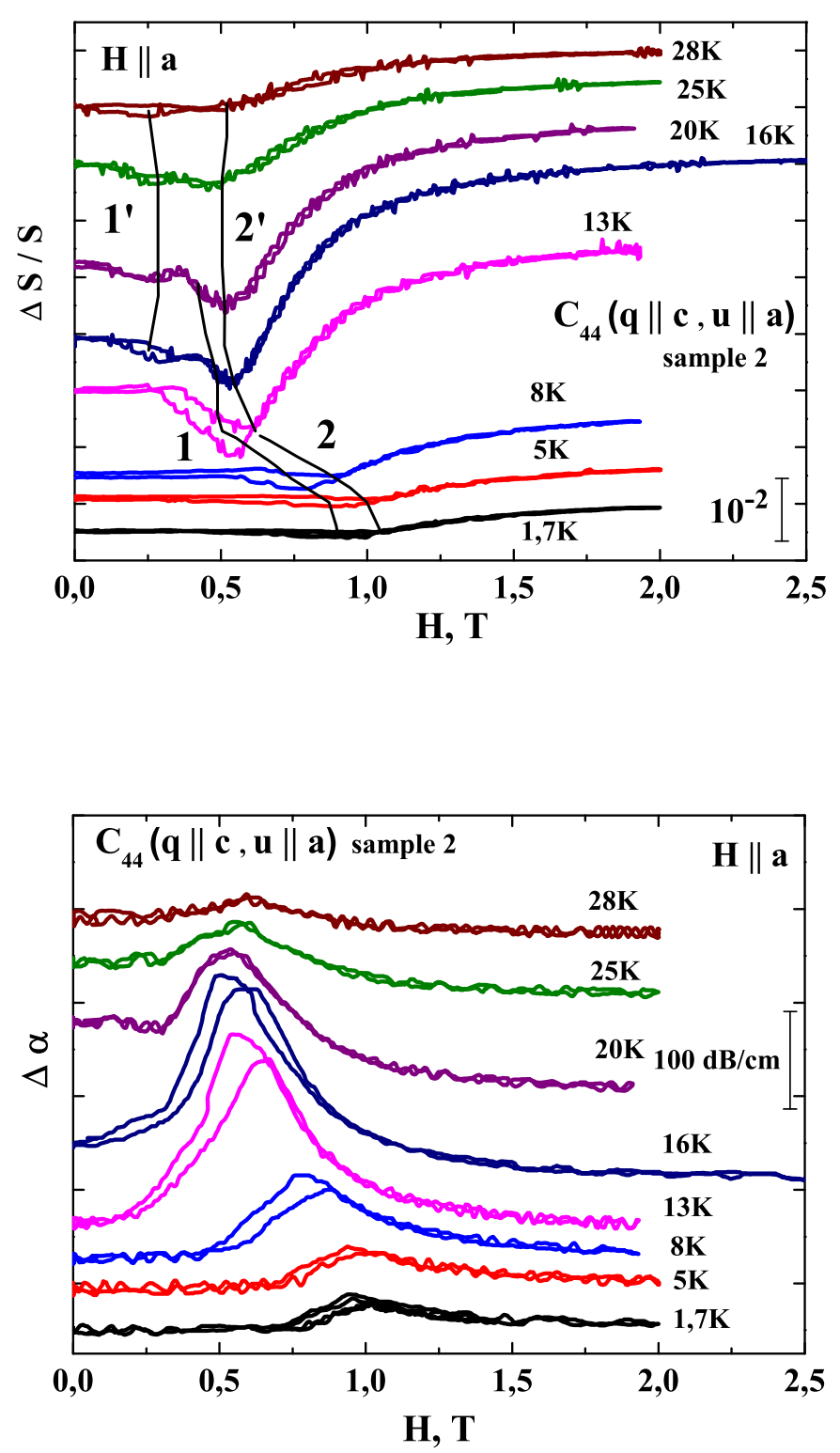

FIG. 6: (Color online) Magnetic field dependence of the velocity (a) and attenuation (b) of the acoustic mode $\mathrm{C}_{44}(\mathbf{q}\|\mathbf{c}, \mathbf{u}\| \mathbf{a})$ at various temperatures in the magnetic field $\mathbf{H} \| \mathbf{a}$. 

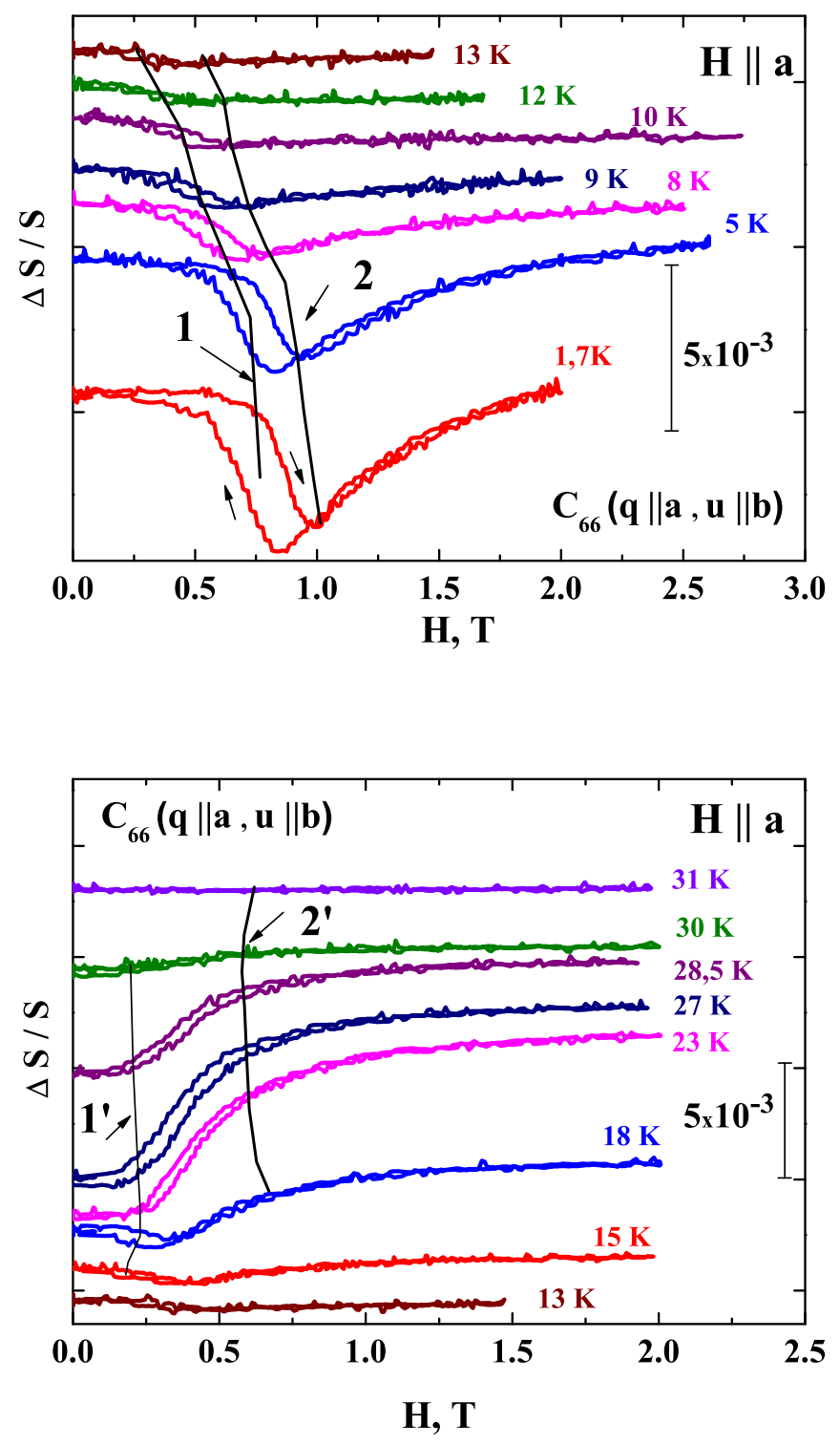

FIG. 7: (Color online) Magnetic field dependence of the velocity of the acoustic mode $\mathrm{C}_{66}(\mathbf{q} \| \mathbf{a}$, $\mathbf{u} \| \mathbf{b}$ ) at temperatures from $1.7 \mathrm{~K}$ till $13 \mathrm{~K}$ (a) and from $13 \mathrm{~K}$ till $31 \mathrm{~K}$ (b) in the magnetic field H $\|$ a. 

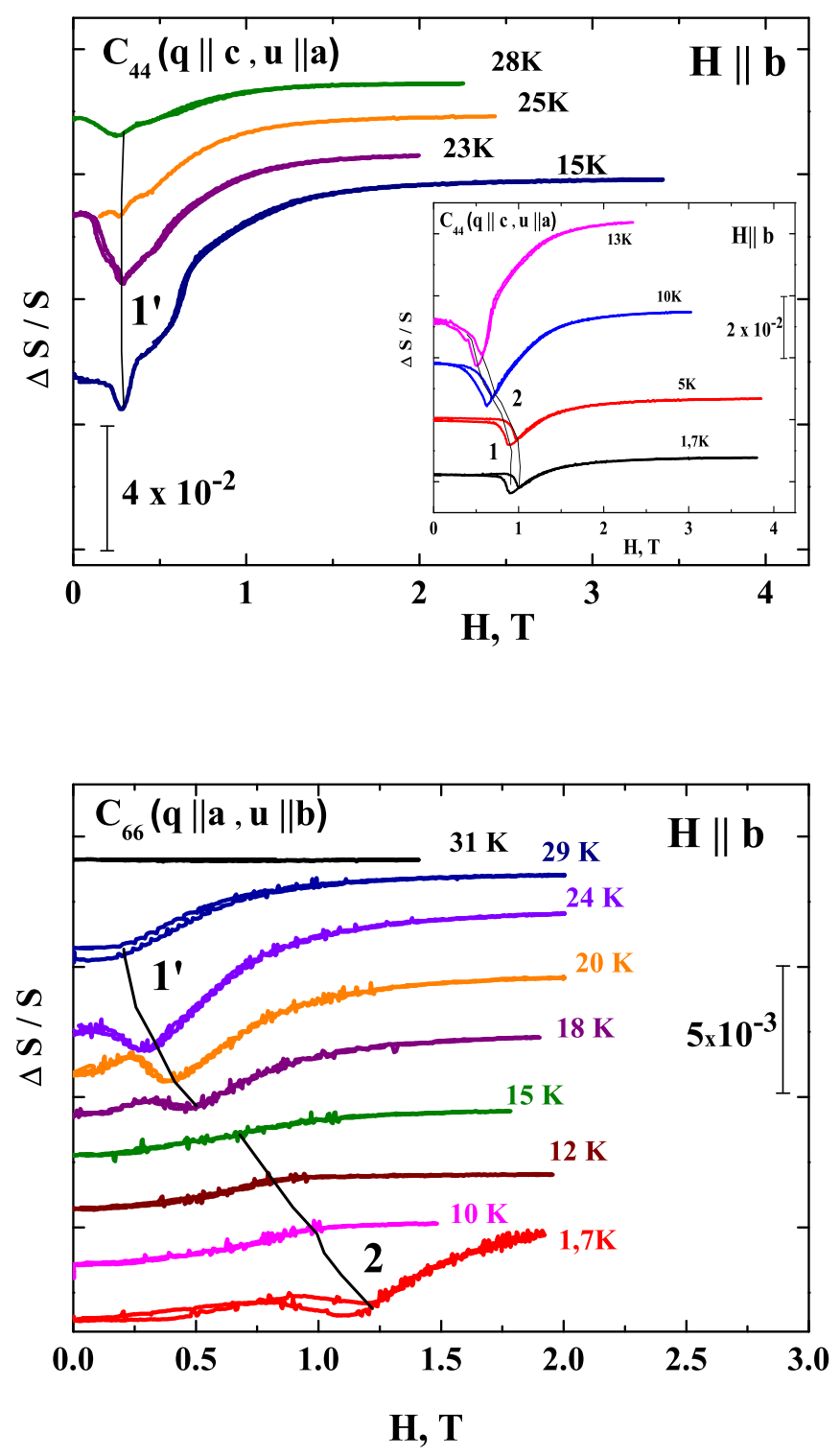

FIG. 8: (Color online) Magnetic field dependences of the velocities of the acoustic modes $\mathrm{C}_{44}$ $(\mathbf{q}\|\mathbf{c}, \mathbf{u}\| \mathbf{a})(\mathrm{a})$ and $\mathrm{C}_{66}(\mathbf{q}\|\mathbf{a}, \mathbf{u}\| \mathbf{b})(\mathrm{b})$ at various temperatures in the magnetic field $\mathbf{H} \| \mathbf{b}$. 

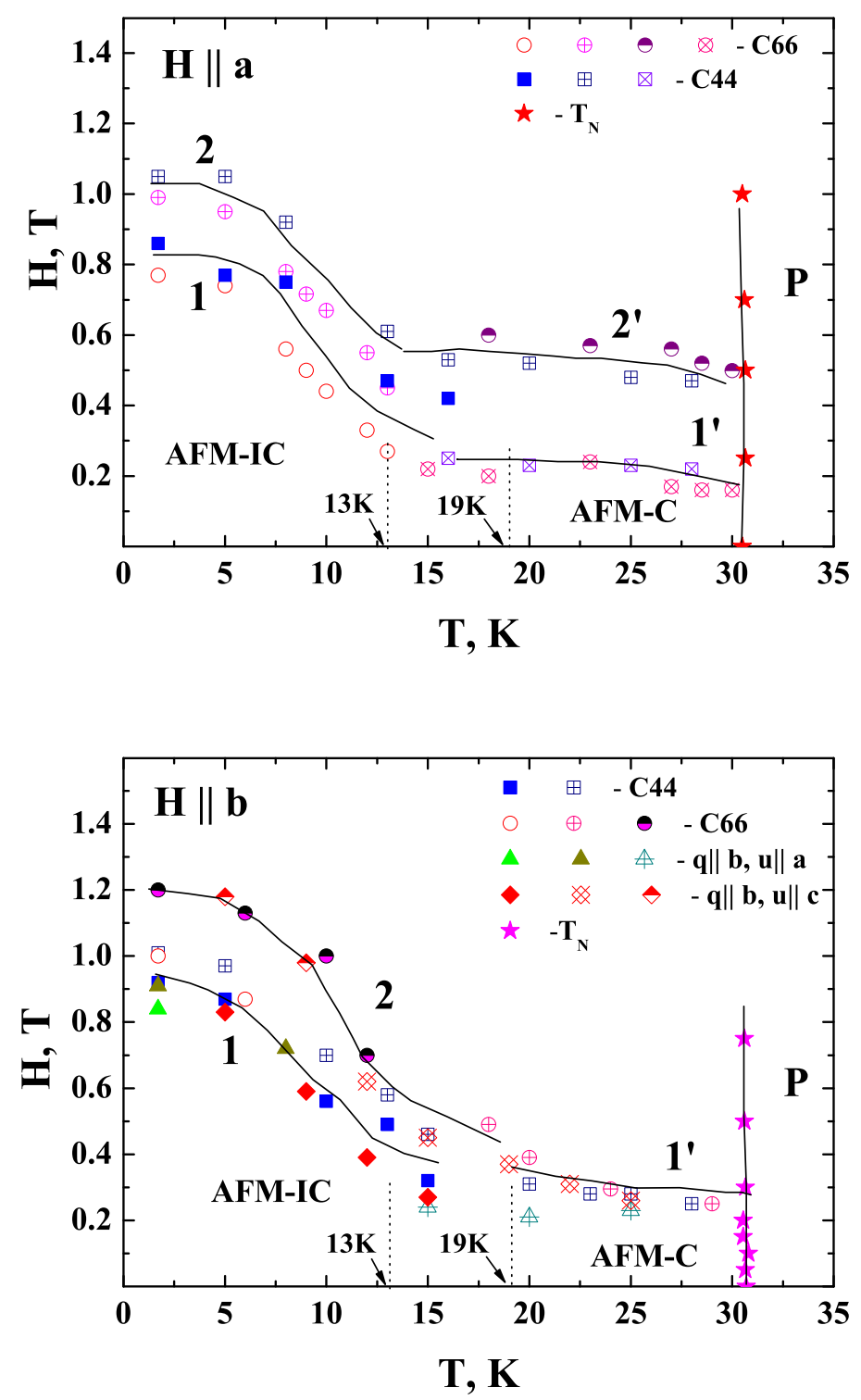

FIG. 9: (Color online) Phase $H-T$ diagrams of $\mathrm{NdFe}_{3}\left(\mathrm{BO}_{3}\right)_{4}$ for $\mathbf{H} \| \mathbf{a}(\mathrm{a})$, and $\mathbf{H} \| \mathbf{b}$ (b). The symbols on diagrams are related to the positions of features in the velocities and attenuations of transverse acoustic modes. Lines are guides to eye. 

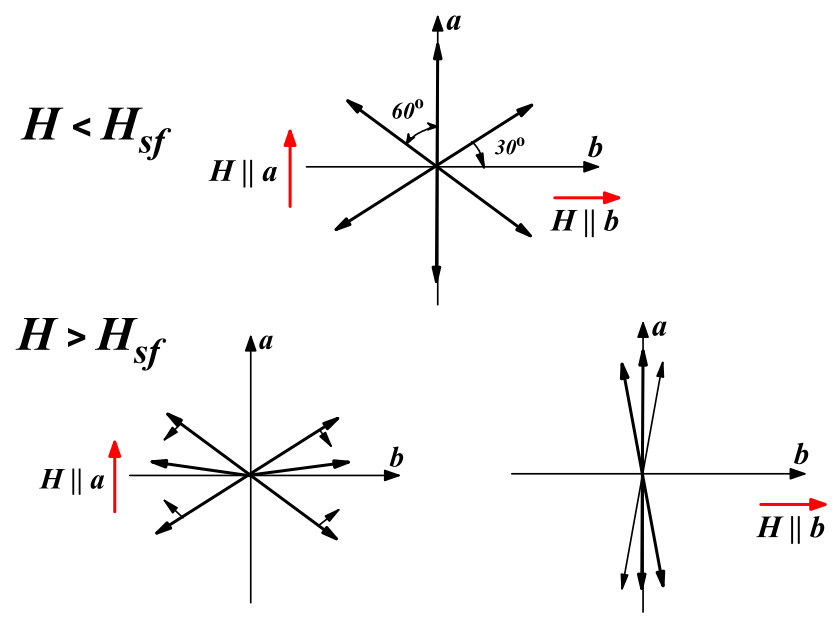

FIG. 10: (Color online) The illustration of the model of the spin-flop transition in the collinear antiferromagnetic phase, the idea is taken from [8]. 